\title{
Análisis de conflictos socioambientales en el parque natural de Las Lagunas de Ruidera. \\ La influencia de la agricultura de regadío sobre el turismo.
}

\author{
María Luz Vázquez Rosa \\ Universidad de Alicante \\ m luzvr88@hotmail.com
}

\section{RESUMEN}

Se presenta el análisis de intereses en el Parque Natural de las Lagunas de Ruidera y los conflictos socioambientales que surgen entre los mismos. Especialmente se tratan los conflictos que aparecen por el agua, por ser uno de sus valores más importante e imprescindible, tanto para su conservación como para el turismo, por la gran afluencia de visitantes que recibe atraídos por este recurso. También es un elemento muy valorado por el resto de actores interesados en el espacio y, sobre todo, para la agricultura de regadío. En este sentido, se hace un análisis más exhaustivo de la influencia que tiene la agricultura de regadío sobre el turismo y los conflictos que surgen por el agua entre estas dos actividades. Finalmente, se realiza un balance de las propuestas existentes para la solución de esos conflictos, destinado a la compatibilidad entre actividades económicas y entre éstas con la conservación del espacio y, por tanto, tendentes a la sostenibilidad del mismo.

Palabras clave: Espacios Naturales Protegidos, partes interesadas, conflictos socioambientales. 


\title{
Social and environmental conflicts analysis in the natural park Las Lagunas de Ruidera. \\ The influence of irrigation agriculture on tourism.
}

\author{
María Luz Vázquez Rosa \\ University of Alicante \\ m luzvr88@hotmail.com
}

\begin{abstract}
The social and environmental conflicts that arise among different interest groups in the Natural Park Lagunas de Ruidera are analyzed in this study presented. The conflicts over water resources are specifically treated. Water is one of their most important values and its conservation its essential for tourism because the park receives a large number of visitors attracted by water. It is also very valued by the other actors interested in area and, especially for irrigated agriculture. In this sense, we carry out a more exhaustive analysis of the influence of irrigated agriculture on tourism and the conflicts over water between these two activities. Finally, we present an evaluation of the existing proposals for resolving these conflicts that try to get the compatibility between economic activities and, between this and the conservation of the area and, therefore, its sustainability.
\end{abstract}

Key words: Protected Natural Areas, stakeholders, social and environmental conflicts. 


\section{INTRODUCCIÓN.}

El Parque Natural de las Lagunas de Ruidera se sitúa al sur de la Comunidad de Castilla-La Mancha, entre las provincias de Ciudad Real y Albacete. Ruidera es el único núcleo de población dentro del parque, pero éste también está formado por los términos municipales de Argamasilla de Alba, Villahermosa y Alhambra en Ciudad Real y Ossa de Montiel en Albacete. Fue declarado Parque Natural el 13 de julio de 1979, con una superficie de 3.772 hectáreas.

Se sitúa en un espacio natural, pero también social (Baigorri, 1999; Corraliza, García y Valero, 2001). Como tal, en él se producen una serie de relaciones entre numerosos actores: agricultores, empresarios turísticos, Junta Rectora del Parque, administraciones públicas, grupos ecologistas, etc., que mantienen diferentes posturas e intereses, tanto de carácter económico o no, que dan lugar a la aparición de conflictos. A estos diferentes intereses se unen las restricciones que conlleva la declaración de protección del espacio que genera una serie de conflictos por el establecimiento de unos límites de uso y aprovechamiento que afectan de manera especial al sector turístico.

Este estudio se divide en cinco epígrafes. El primero trata los conflictos socioambientales teóricos que mejor se adaptan al caso de estudio. Posteriormente se concretan los objetivos del trabajo y la metodología empleada para conseguir los mismos. En el cuarto epígrafe se presenta el anàlisis y los resultados obtenidos. Dentro de este apartado, en primer lugar se hace una gradación de la valoración que dan los actores a la conservación de los recursos naturales del parque para situar a los mismos en una posición con respecto a la conservación y facilitar los posteriores análisis de las relaciones que se mantienen entre actores y los conflictos existentes. En segundo lugar, el recurso natural y atractivo principal del Parque Natural de las Lagunas de Ruidera es el agua, junto con los materiales calizos, responsables de su formación. Por ello, especialmente se tratan los conflictos que aparecen por el agua, por ser uno de sus valores más importante e imprescindible tanto para su conservación, como para el turismo y la agricultura de regadío. Así, se hace un análisis más exhaustivo de la influencia que tiene la agricultura de regadío sobre el turismo y los conflictos que surgen por el agua entre estas dos actividades. Además, se trata en profundidad otro de los conflictos más importantes identificado en el Parque, la gestión del mismo. Este conflicto enfrenta a la Dirección del Parque con el resto de partes interesadas en el espacio. Se trata de un conflicto que tiene su origen en las diferentes valoraciones sobre la conservación y aprovechamiento de los recursos naturales. Pero también con respecto a la adecuación del espacio para su uso público $y$, por tanto, también turístico. Dentro del conflicto por la gestión, se profundiza en el grado de participación en la toma de decisiones, que explica muchas de las razones por las que surge el mismo. Para finalizar con este apartado, se realiza un balance de las propuestas existentes para la solución de esos conflictos. Por último, se indican las conclusiones derivadas del estudio. 


\section{MARCO TEÓRICO}

\subsection{Conflictos socioambientales}

El principal objetivo de la gestión de los parques naturales es la consecución de un equilibrio entre la conservación de sus recursos naturales con el desarrollo socioeconómico y el uso público. Pero en ese equilibrio es donde residen la mayoría de conflictos por la dificultad de establecer los límites entre uso y conservación. A este se une la dificultad de gestionar un espacio en el que existen multitud de agentes con diferentes intereses que, lógicamente, conlleva la aparición de conflictos sociales (Pulido, 2010).

La mayoría de conflictos se relacionan con el uso y gestión de los recursos naturales, por lo que sería más correcto hablar de conflictos socioambientales ${ }^{1}$.

\subsubsection{Tipos de conflictos socioambientales.}

A continuación se proponen dos de las clasificaciones más útiles para la realización del proyecto. La primera atiende a las relaciones que se producen entre actores, destacando los siguientes conflictos:

- Conflictos entre conservacionistas y productivistas: se producen por valoraciones diferentes sobre la conservación. Los primeros adoptan una postura biocentrista, que da un valor a la naturaleza totalmente independiente del que pueda darle el ser humano; en otras palabras, un valor intrínseco (Aledo y Domínguez, 2001). Los productivistas defienden el antropocentrismo más extremo adoptando una posición utilitarista por cuanto la naturaleza para el ser humano sólo tiene valor si puede serle productiva; un valor extrínseco y utilitarista. En la realidad, esta clasificación no es tan tajante, pues existen multitud de posiciones intermedias.

- Conflictos entre actores o actividades económicas: normalmente se producen por la incompatibilidad de usos productivos entre actores con diferentes intereses en el espacio y sus recursos naturales. Esto puede ser más problemático cuando existe una incompatibilidad de intereses por el uso de un mismo recurso.

- Conflictos entre instituciones públicas y actores sociales y económicos: vienen provocados por la regulación de usos, planeamiento de suelo, medidas de protección, prohibición de actividades, etc., por parte de la administración. Estas actuaciones son las que en mayor o menor medida provocan conflictos por la restricción de actuaciones y limites a la explotación de los recursos naturales.

La segunda de las clasificaciones distingue entre los conflictos por el acceso, uso y conservación de los recursos hídricos. Este tipo de conflictos giran en torno a tres cuestiones básicas sobre el agua: la cantidad, la disponibilidad y la calidad (Wolf, Kramer, Carius, y

\footnotetext{
${ }^{1}$ Entendemos por tales la contraposición o incompatibilidad de posturas, en relación al acceso, uso y significado de los recursos naturales y a diferentes posturas sobre cómo se define la degradación ambiental (Buckles y Rusnak, 1999; Borel, 1999; Adams, 2003).
} 
Dabelko, 2005). Este último atributo, podríamos decir que el más importante, determina que el agua, en mayor o menor cantidad, pueda ser utilizada o disfrutada por los usuarios de la misma. Además, nos encontramos en ENP con una gran cantidad de especies singulares y endémicas que dependen, además de la cantidad, de la calidad del agua para poder sobrevivir. Atendiendo a aquellos espacios en los que está permitido el baño, la calidad del agua es fundamental. Si contamos con aguas contaminadas, la salud de los bañistas puede verse perjudicada, traduciéndose en una mala imagen para el espacio, reducción del número de visitantes y, por tanto, del volumen de ingresos al sector turístico. Efectos en la salud, también pueden producirse en el agua para consumo humano, actividades industriales y para la agricultura. Por tanto, una mala calidad del agua puede ser fuente de conflictos entre los responsables de su contaminación y los afectados.

Por otra parte, la cantidad del agua debe ser gestionada cuando en un espacio existen múltiples actividades que tienen a esta como recurso principal. Si no, se entraría en una disputa en la que la actividad de mayor poder en la zona sería la más beneficiada en detrimento del resto. A esto se une que en los espacios naturales húmedos, la principal atracción con la que cuentan es el disfrute del agua, no sólo del baño sino también de su contemplación. Un humedal que sufra escasez de agua no será atractivo para la visita. De nuevo se muestra aquí un posible conflicto entre los actores que han contribuido o son responsables de la sobreexplotación de los recursos hídricos y el sector turístico.

Por último, los conflictos por la disponibilidad del agua vienen en su mayoría causados por el coste de extracción y de su uso inmediato. Como ejemplos se puede señalar el aprovechamiento del agua por la agricultura de regadío, el consumo humano o el uso para la vivienda. Para ello es necesario que se realicen perforaciones del subsuelo que pueden suponer un sobre coste para otros usuarios si se producen de manera ilegal y por encima de la cantidad que tienen asignada en su caso. Esta mala conducta provoca que el resto de usuarios tengan que realizar obras de perforación más profundas para poder extraerla. Esto supone un sobre coste, además de la sobreexplotación y posible salinización del acuífero.

Todos estos conflictos, se pueden interpretar a la luz de la conocida Tragedia de los Comunes (Hardin, 1968). Esta teoría defiende que la propiedad común o el libre acceso a los recursos, conlleva la sobrexplotación del recurso y su degradación hasta la desaparición total en perjuicio del bien común. Se produce porque los usuarios del recurso mantienen un aprovechamiento en beneficio propio, sin tener en cuenta el bien común. Esto ocurre cuando un recurso no tiene ningún tipo de gestión o límite de uso, por lo que el acceso es libre. Así, ningún usuario puede ser excluido de su uso pero depende del uso de los demás para llevar a cabo el propio. Las soluciones que se proponen para un desarrollo sostenible del recurso, son variadas, desde la gestión por todos los usuarios, pasando por su privatización, hasta el establecimiento de una autoridad superior que establezca las reglas que rigen su aprovechamiento. 


\section{OBJETIVOS}

El objetivo principal del estudio es conocer los conflictos socioambientales existentes en el Parque Natural de las Lagunas de Ruidera. Para poder alcanzarlo se establecen varios objetivos específicos que van desde la identificación de los conflictos en el espacio, a los actores representativos de los mismos, pasando por un conocimiento más exhaustivo de la problemática socioambiental a través de la opinión y valoración tanto de la literatura existente como de los actores identificados para analizar el grado de conflicto entre dichos actores y sus intereses, valores y actitudes con respecto a la conservación del espacio natural. También se propone como objetivo específico identificar y analizar los conflictos por el agua entre la agricultura y el turismo, para conocer la influencia de la primera sobre la segunda y también sobre la conservación. Para finalizar, se plantea reflexionar y discutir las propuestas dirigidas a la mitigación de los conflictos existentes por el agua en las Lagunas de Ruidera, por parte de los actores identificados.

\section{METOdologíA.}

En consonancia con los objetivos de la investigación, se ha empleado una metodología cualitativa basada principalmente en dos técnicas: la entrevista en profundidad y la observación participante. A estas dos técnicas, se suman el estudio del espacio y del tema a través de la revisión bibliográfica, la documentación y la recogida de datos secundarios. A continuación se especifican los objetivos a conseguir con cada una:

Los objetivos de la revisión bibliográfica son:

- Identificar y conocer los conflictos más importantes en los espacios naturales protegidos y, en concreto, los conflictos existentes en el Parque Natural de Lagunas de Ruidera.

- Identificar los actores con intereses en el parque, involucrados en los conflictos que se producen.

La recogida de datos secundarios se emplea para:

- Servir de apoyo y ejemplo para la investigación

- Ayudar a comprender y profundizar mejor en el espacio objeto de estudio.

La observación participante tiene como objeto:

- La revisión y crítica de lo investigado para poder reafirmar valoraciones y afirmaciones ya expresadas por otros autores o descartarlas del estudio.

- La integración en el espacio, su comunidad, acontecimientos, etc., que puede ayudar a comprenderlo mejor.

- Adoptar una postura externa de investigador ajeno al lugar, que brinda la objetividad para poder analizar la información recabada a través de esta técnica. 
La entrevista en profundidad se lleva a cabo con aquellos actores interesados, identificados previa y posteriormente por las técnicas escogidas. La entrevista en profundidad tiene como objeto obtener la información que dé respuesta a los dos primeros objetivos del estudio, que eran:

1. Identificar los conflictos socioambientales existentes en el parque.

o Conocer los conflictos del Parque a través de la opinión de los distintos actores interesados.

o Identificar los actores representativos de los conflictos.

o Analizar el grado de conflicto entre dichos actores y sus intereses, valores y actitudes con respecto a la conservación del espacio natural.

2. Identificar y analizar los conflictos por el agua entre la agricultura y el turismo.

El trabajo de campo se desarrolló desde abril hasta agosto de 2011, con diferente intensidad. En una primera etapa se realizó una toma de contacto con el lugar como investigador del mismo. De este modo, como parte de la observación participante, se estableció contacto con el escenario y se organizaron las posteriores visitas al mismo, tanto para su análisis como para realizar las entrevistas pertinentes. Éstas se llevaron a cabo desde la última semana de julio hasta la tercera de agosto. Los resultados del análisis de entrevistas que se presentan a continuación, son fruto de la interpretación de las mismas, apoyada por un proceso previo de categorización y análisis.

\section{ANÁLISIS Y RESULTADOS.}

\subsection{Conflicto entre conservacionistas y productivistas.}

El gráfico está elaborado mediante el análisis de los comentarios de los actores entrevistados, en función de la valoración de los ámbitos económico y conservacionista con el espacio. Para simplificar se han dividido en 6 categorías que van desde las posturas más extremas, biocentrismo y antropocentrismo, tomando una media y débil para ambas. Así el resultado obtenido para cada actor se encuentra reflejado por el número que le corresponde, que atiende al siguiente orden de entrevistas:

1. Alcalde; 2. Empresario Turismo Activo; 3. Concejal de Medio Ambiente; 4. Empresario Alojamiento Camping; 5. Directora-Conservadora; 6. Director Consorcio Alto Guadiana; 7. Representante Ecologistas en Acción; 8. Presidente de la Comunidad de Regantes de Aguas Privadas del Campo de Montiel; 9. AgricultorGanadero; 10. Empresario Restauración; 11. Encargado Albergue Juvenil.

Gráfico 1. Valoración de la conservación por los actores del 
Parque Natural de las Lagunas de Ruidera

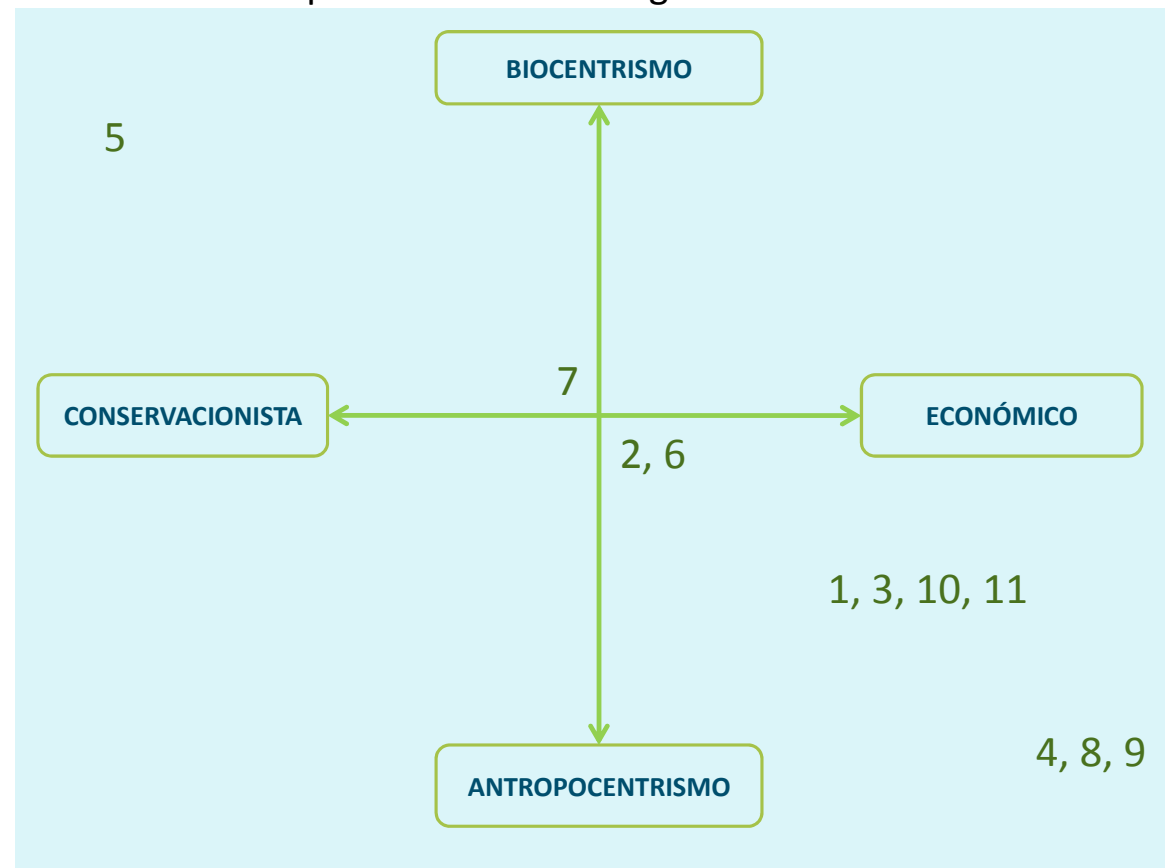

Fuente: Elaboración propia

Este análisis nos presenta un conflicto entre aquellos que defienden que la conservación debe anteponerse a cualquier actividad o uso público (5), y aquellos que creen que sin el último, el espacio no presentaría ningún interés y se degradaría $(4,8$ y 9). Sin embargo, también se observa que existen posturas menos enfrentadas que abogan por esa compatibilidad tan deseada entre conservación y uso público $(7,2,6,1,3,10,11)$.

En relación con lo anterior, existe una relación negativa entre los actores con intereses económicos y los actores más conservacionistas. Hay un alto grado de enfrentamiento entre el resto de actores con la dirección del ENP por la falta de participación y las restricciones. Pero también existe una mala relación entre el sector turístico y los agricultores por la lucha de intereses que mantienen sobre el agua, como se explica más adelante.

\subsection{Conflictos por el acceso, uso y conservación de los recursos hídricos en el Parque Natural de las Lagunas de Ruidera.}

En la actualidad los recursos hídricos del Parque, en general, presentan un buen estado, sobre todo en cantidad y disponibilidad para todos los usuarios o actores interesados en la gestión, aprovechamiento y conservación del agua. Sin embargo, se trata de un análisis superfluo pues también se está de acuerdo en que los conflictos por el agua son pasados, presentes y futuros ya que no se han adoptado las medidas necesarias para su eliminación, sino más bien se han ocultado en acciones de dudosa o nula eficacia y que no han resuelto los conflictos existentes por el agua $(2,4,6,7,11)$ 
En la cantidad no existe un conflicto manifiesto, gracias al ciclo húmedo por el que actualmente se está atravesando. Si bien, el conflicto se encuentra presente para varios autores, quienes opinan que no se ha terminado de resolver. En el futuro esto puede provocar problemas de cantidad de agua que enfrenten a actores que defiendan la conservación del parque, ya sea por su interés ambiental o económico contra la actividad agrícola, por ser ésta la mayor consumidora de los recursos hídricos en la cabecera del parque $y$, por tanto, la que afecta directamente a la cantidad que entra en el mismo. La falta de control total o eliminación de los regadíos comprometen el futuro del parque, entre otros aspectos, de forma permanente mientras persistan los mismos $(2,4,5,7,10,11)$.

Por otro lado, el sector agrícola expone que el conflicto por la cantidad de agua es inexistente. Sin embargo, se encuentra una contradicción al abogar por la compatibilidad entre la conservación y su uso del agua, como si de verdad existiera una disputa por el agua entre la agricultura de regadío y la conservación del parque. Dicha compatibilidad parece no importar siempre y cuando se pueda continuar con la actividad agraria pues se adopta una posición de poder con respecto al uso del agua y se apuesta por la descatalogación de terrenos protegidos si la economía lo requiere.

En cuanto a la calidad del agua, se identifica como un conflicto actual. A pesar de que la misma no presenta niveles de contaminación perjudiciales para el baño o la conservación de las especies, sí presenta problemas para el consumo humano no siendo potable en el municipio de Ossa de Montiel. El agua que llega al parque presenta un alto porcentaje de nitratos $(1,2,4,5,6,10,11)$. Todos los actores coinciden en que al contenido de nitratos se suma la falta de depuración de las aguas residuales de las construcciones situadas dentro del parque que continúan contaminando las aguas. Esto se debe a que el proyecto de Saneamiento Integral se encuentra incompleto, pues la obra principal está realizada. La depuradora situada en el municipio de Ruidera se encuentra en funcionamiento, pero no las conexiones de los edificios situados dentro del espacio protegido.

La disponibilidad de agua para todos los actores interesados es buena debido a que actualmente hay agua suficiente para todos y no se aprecia como un conflicto directo. Se trata más bien de un conflicto pasado sin resolver que, actualmente, se encuentra oculto por las abundantes precipitaciones del pasado y presente año. Por tanto, si se identifica como conflicto el hecho de que la disponibilidad del agua para la agricultura de regadío que se sitúa en la cabecera de las Lagunas compromete la disponibilidad de la misma para el parque, y de esta forma para su conservación y para el turismo, tan dependiente de este recurso $(2,4,5$, $7,11)$.

En resumen, los conflictos por el agua son un tema actual a tratar en el espacio porque se siguen adoptando posturas enfrentadas en cuanto al acceso, uso y conservación de los recursos hídricos. Los diferentes intereses sobre este recurso, a pesar de su buen estado de conservación actual, y la ineficaz puesta en marcha de medidas para regular las extracciones y contaminación por la agricultura, así como la depuración de las construcciones, mantienen vivos los conflictos por los recursos hídricos. 


\subsection{Conflictos entre la agricultura y el turismo.}

Los conflictos entre estas dos actividades están muy estrechamente relacionados con los conflictos entre la agricultura y la conservación del Parque Natural, por la relación tan estrecha que hay entre el turismo y la conservación del espacio. Así, se pueden señalar conflictos en la cantidad del agua de extracción para la agricultura, que al mismo tiempo que afecta a la conservación del espacio afecta al turismo por la reducción, o incluso desaparición, de zonas de baño. Por tanto, los conflictos entre la agricultura y el turismo surgen sobre todo por la cantidad y la disponibilidad de agua. Sigue siendo un problema la falta de un límite de extracción para el regadío, acorde con la renovación del acuífero y que no provoque la desecación de las Lagunas. A pesar de que la calidad es un aspecto fundamental de la misma, no se identifica como un posible conflicto en este sentido. No ocurre lo mismo con la conservación del parque, o cuando se pregunta por separado sobre este atributo.

\subsection{Conflictos entre la gestión del espacio y el uso turístico.}

Este enfrentamiento se debe a la pretensión del sector turístico por querer llevar a cabo acciones en el espacio que aumenten su volumen de negocio y que conllevan, en muchas ocasiones, la degradación de los valores naturales del Parque. También por la mala gestión del espacio que se ha mantenido a lo largo de los años y que ha provocado una situación actual de escasa adecuación de infraestructuras, de oferta turística, falta de promoción para la desestacionalización del parque, escasez de servicios de atención al visitante y de limpieza y vigilancia, que repercuten negativamente en el sector turístico.

La situación anterior está muy relacionada con la inexistencia y falta de voluntad por parte de la Dirección del Parque de diseñar un proceso de participación activa en la toma de decisiones sobre la gestión.

\subsection{Propuestas para la mitigación de los conflictos existentes por el agua.}

En resumen, por parte de los agricultores se pretende que las extracciones se regulen en función de la pluviometría de cada período sin contemplar la reducción de sus derechos. Esta propuesta supondría un paso atrás en la gestión del agua que aceleraría los períodos de escasez si no se establecen unos límites de extracción coherentes con el resto del sistema y que aseguren la renovación del recurso.

El resto de actores, en su mayoría, son tolerantes con la agricultura pero proponen el establecimiento de unas cantidades de agua que no afecten al nivel de las lagunas, así como continuar con los controles de nitratos para evitar que los niveles de contaminación superen el límite establecido.

En cuanto a la depuración de las construcciones del parque, se propone la conexión de las mismas a la red de colectores lo antes posible para su depuración correcta.

\subsection{Propuestas para la mejora de la gestión turística del parque.}


En primer lugar, se propone una gestión conjunta entre la Dirección y los ayuntamientos $(1,2,3$ y 4), con objeto de posibilitar la participación activa de todos los actores interesados, propuesta con la que están de acuerdo todos los actores excepto la Dirección que no se pronuncia al respecto. Se plantea la modificación del actual PRUG que se encuentra desfasado, un Plan de Ordenación del Territorio y la realización de un Plan de Uso Público. Este último, necesario en un espacio natural e imprescindible en este espacio natural en concreto por el gran uso público y turístico con el que cuenta $(1,3$ y 7$)$.

Así también, la mayoría de actores se muestra favorable a la adecuación de las infraestructuras, para hacer frente a la masificación, y la realización un Plan de Promoción del Parque que favorezca la desestacionalización del mismo.

Otras propuestas son la protección de las barreras travertínicas (7) y un aumento tanto de la formación de los recursos humanos como de la calidad de la oferta $(6,7,2$ y 11$)$.

\section{CONCLUSIONES}

En definitiva, los conflictos por el agua en el Parque Natural de las Lagunas de Ruidera no se han terminado de resolver, a pesar de que el buen ciclo hidrológico por el que se está atravesando hace que tanto la calidad, como la cantidad y la disponibilidad para todos los usuarios sea buena, en general. Sin embargo, el hecho de no haber adoptado medidas definitivas para su paliación puede volver a provocar o agravar los mismos efectos que se produjeron en el pasado. Frente a este posible escenario de futuro, sólo puede tomarse una dirección, que va en el sentido de finalizar aquellos planes y actuaciones que se pusieron en marcha para solucionar estos problemas y que, por unos $u$ otros motivos, no han terminado de llevarse a la práctica. Ante la dificultad de concluir con esas actuaciones, se podrían adoptar otras con el mismo fin. En este sentido, tanto los ayuntamientos, como la Dirección del Parque y el sector turístico tienen mucho que decir. Estos grupos deben de hacer presión para que la actividad agrícola no perjudique su propia actividad, sino que sea regulada para que desaparezca el conflicto por el aprovechamiento del agua, así como que se disminuyan las cantidades de nitratos utilizados. Por último, el sector turístico, debe tomar una actitud más reivindicativa frente a la administración para que lo antes posible se solucione la falta de depuración.

En cuanto a la gestión turística, es fundamental que exista un proceso de participación activa en aquellas acciones como la adecuación de las infraestructuras de acogida, de la oferta, etc. Sin embargo, existen otras tareas como la conservación y protección que deben ser desempeñadas por técnicos especialistas. En estas tareas no cabe la participación, pues cada actor interesado en el espacio primaría sus intereses por encima de los intereses del resto de actores, incluso de la conservación. Cabe recordar que una de las soluciones a la gestión de conflictos planteada en el marco teórico era el establecimiento de una autoridad superior que regulase el aprovechamiento de los recursos naturales para evitar su degradación por el interés particular. En este espacio este papel sería el representado por la Dirección-Conservación del Parque que adopta una postura conservacionista para evitar el deterioro de esos recursos. Se puede decir entonces que, en 
este caso concreto y mediante esa autoridad, el objetivo de conservación se cumple. No podría llevarse a cabo la gestión privada ni conjunta de los recursos al existir posturas tan diferentes sobre la conservación.

Así mismo se debe hacer entender a la Dirección, hasta el momento con una mayor preocupación por las acciones de regulación y control, que la conservación del medio también puede alcanzarse mediante la cooperación de todas las partes interesadas "para que no le maten de amor" o "muera por su belleza", recordando una cita parafraseada por la Directora del Parque. Ese morir por amor, no sólo puede interpretarse como el uso intensivo del espacio que lo termine degradando. También puede interpretarse como un amor "celoso" que lleve a convertir el espacio en un museo que cuando cierra sus puertas al público queda inerte, sin más futuro que el abandono de la actividad económica y del cuidado de su población local y sin más futuro que su extinción. Para el turismo la conservación de los recursos naturales ha de ser el principal objetivo pues es crucial para su actividad. Del mismo modo que el principal objetivo, y a la vez reto, de la gestión de un parque natural es encontrar ese equilibrio entre la conservación de sus recursos naturales, con el desarrollo socioeconómico y el uso público.

\section{REFERENCIAS BIBLIOGRÀFICAS}

ADAMS, W. (2003). "Managing Tragedies: Understanding Conflict over Common Pool Resources", Science, 302 (5652), 1915-1916.

ALEDO, A., DOMínGUEZ, J.A. (2001). Sociología Ambiental. Granada: Grupo Editorial Universitario.

BAIGORRI, A. (1999). "De la naturaleza social de la Naturaleza. Ecología, regadíos y protección ambiental". En Publicado en PARDO, M. ed., Sociología Medioambiental. Estado de la cuestión. Madrid: Fundación de los Ríos.

BOREL, R. (1999). Conflictos socioambientales en América Latina: un intento de tipología, mapeo y análisis comparado de casos. San José, Costa Rica: Cedarena.

BUCKLES, D. y RUSNAK, G. (1999). "Conflict and Collaboration in Natural Resource Management", en D. BUCKLES (ed.), Cultivating Peace: Conflict and Collaboration in Natural Resource Management. Ottawa: Institute for Development Research Cooperation.

CORRALIZA. J.A., GARCÍA. J. y VALERO. E. (2001). Los Parques Naturales en España: conservación y disfrute. Madrid: Fundación Alfonso Martín Escudero.

HARDIN, G. (1968). "The Tragedy of the Commons". Science, 162, (3859), 1243-1248.

PULIDO, J.I. (2010). "Las partes interesadas en la gestión turística de los parques naturales andaluces. Identificación de interrelaciones e intereses". Revista de Estudios Regionales, 88, 147-175.

PULIDO, J.I. y MERINERO, R. (2009). “Desarrollo turístico y dinámica relacional. Metodología de análisis para la gestión activa de destinos turísticos". Cuadernos de Turismo, 23, 173-193.

WOLF, A.; KRAMER, A.; CARIUS, A. y DABELKO, G. (2005). "Gestionando conflictos por el agua y cooperación". Publicado en: RENNER, M.; FRENCH, H. y ASSADOURIAN, 
E. (dir.). La Situación del Mundo 2005: Redefiniendo la seguridad mundial. Informe Anual del Worldwatch Insttute sobre el progreso hacia una sociedad sostenible, Barcelona: Icaria; Centro de Investigación para la Paz, 155-178.

\section{Nota:}

El presente artículo es un extracto del trabajo presentado por la autora como Trabajo de Fin de Máster en Dirección y Planificación del Turismo de la Universidad de Alicante: http://rua.ua.es/dspace/handle/10045/19452 